\title{
Observation of the two-channel Kondo effect
}

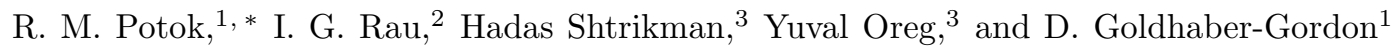 \\ ${ }^{1}$ Department of Physics, Stanford University, Stanford, California 94305 \\ ${ }^{2}$ Department of Applied Physics, Stanford University, Stanford, California 94305 \\ ${ }^{3}$ Department of Condensed Matter Physics, Weizmann Institute of Science, Rehovot, Israel
}

(Dated: May 6, 2019)

\begin{abstract}
Some of the most intriguing problems in solid state physics arise when the motion of one electron dramatically affects the motion of surrounding electrons. Traditionally, such highly-correlated electron systems have been studied mainly in materials with complex transition metal chemistry. Over the past decade, researchers have learned to confine one or a few electrons within a nanoscale semiconductor "artificial atom", and to understand and control this simple system in exquisite detail. In this Article, we combine such individually well-understood components to create a novel highlycorrelated electron system within a nano-engineered semiconductor structure. We tune the system in situ through a quantum phase transition between two distinct states, one familiar and one subtly new. The boundary between these states is a quantum critical point: the exotic and previously elusive two-channel Kondo state, in which electrons in two reservoirs are entangled through their interaction with a single localized spin.
\end{abstract}

The Kondo effect has become a hallmark of coherent electron transport in a variety of nanostructures ranging from lithographically-defined semiconductors [1] to carbon nanotubes [2] and molecules [3, 4]. Kondo applied the phenomenological Hamiltonian $[5]$

$$
H_{K}=J \vec{s} \cdot \vec{S}+H_{\text {reservoir }}
$$

to describe a magnetic impurity embedded in a host sea of electrons. A localized spin $\vec{S}$ couples antiferromagnetically with strength $J$ to spins $\vec{s}$ of electrons in the surrounding reservoir. $H_{\text {reservoir }}$ represents the free electrons in the reservoir. At temperatures below the Kondo temperature $T_{\mathrm{K}}$, electrons in the reservoir screen the localized spin. The Kondo Hamiltonian was later found to be derivable from the more microscopic Anderson model, which consists of an electron bound to an impurity site in a metal host (Fig. 1(a)). Here, Kondo's antiferromagnetic coupling emerges from tunneling on and off the local site.

Many systems of strongly-interacting particles can be understood in the framework of Landau's Fermi liquid theory, whose basic entities, termed quasiparticles, roll most effects of interactions into changes in particle properties such as mass and energy. Although the Kondo ground state is complex, its excitations can still be described as weakly-interacting quasiparticles. Some of the most intriguing problems in solid state physics arise when this simplification cannot be applied. Examples of such highly-correlated systems include Luttinger liquids, fractional quantum Hall Laughlin liquids, high-temperature superconductors, and the two-channel Kondo system, a novel state studied experimentally in this Article.

In the two-channel Kondo $(2 \mathrm{CK})$ model, introduced 25 years ago by Nozières and Blandin, and independently by Zawadowski [6, 7], a localized spin $\vec{S}$ is antiferromagnetically coupled to two independent reservoirs of electrons according to the Hamiltonian

$$
H_{2 C K}=J_{1} \vec{s}_{1} \cdot \vec{S}+J_{2} \vec{s}_{2} \cdot \vec{S}+H_{\text {reservoirs }} .
$$

The symmetric 2CK state is formed when the two independent channels (or reservoirs) are equally coupled to the magnetic impurity, i.e. $J_{1}=J_{2}$. Each reservoir individually attempts to screen the local spin. Since they cannot both screen the spin, this is an unstable situation, resulting in a new ground state in which the local impurity is only partially screened. Unlike for single-channel Kondo (1CK), in the 2CK state the quasi-particle concept of Fermi liquid theory does not apply: the decay rate for a low energy excitation $(\sim \sqrt{\epsilon})$ is greater than the energy $\epsilon$ of the excitation itself. Stable low-lying excitations must thus be collective [8, 9].

Any difference in channel coupling will force the system away from the non-Fermi liquid 2CK state and toward the $1 \mathrm{CK}$ state associated with the more strongly-coupled reservoir. Although the symmetric $2 \mathrm{CK}$ state might therefore seem difficult to access, it has been invoked to explain remarkable low-energy properties of some heavy Fermion materials 10, 11, 12 and glassy metals 13, 14, 15. However, the connections of these experimental observations to $2 \mathrm{CK}$ theory remain problematic [16], in part because the microscopic electronic structure of the various materials is unclear.

In this Article, we present experimental results on an artificial impurity which is designed to display 2CK. Crucially, we can precisely control the coupling constants $J_{1}$ and $J_{2}$, while maintaining the independence of the two channels. 

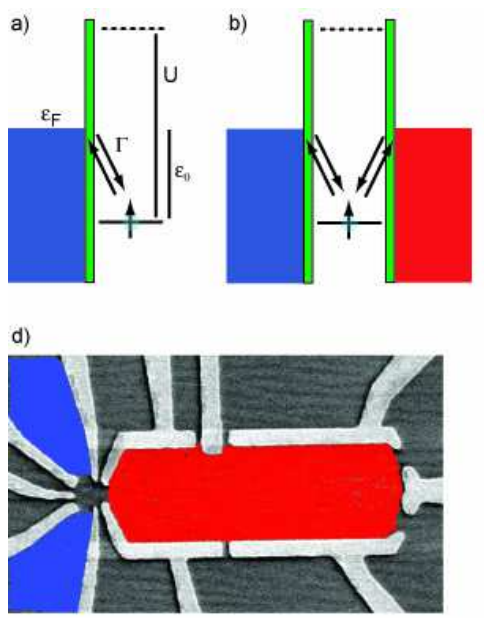

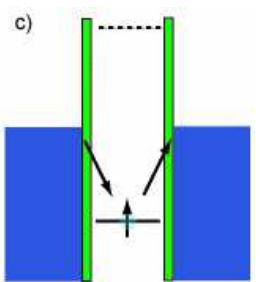

e)

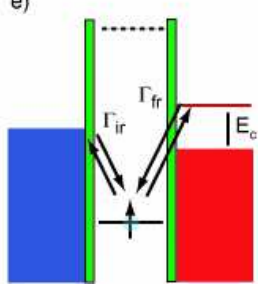

FIG. 1: Single Channel Kondo Effect (1CK). (a) The Anderson model describes a magnetic impurity in a metal as a single spin-degenerate state (right side of green barrier) coupled to a Fermi reservoir of electrons (left). Coulomb interaction $U$ between localized electrons favors having only a single electron in the localized state. The antiferromagnetic coupling $J$ between the localized spin and the reservoir depends on the tunneling rate $\Gamma$, the depth of the level $\epsilon_{0}$, and $U$, according to $\left.J \sim \Gamma U /\left(\epsilon_{0}\left(\epsilon_{0}+U\right)\right) 21\right]$. At low temperature, high-order tunneling processes between the local state and the Fermi reservoirs coherently add together to screen the localized electron spin. (b) Two Channel Kondo Effect (2CK). A localized electron is now coupled to two independent Fermi reservoirs (blue and red). If the two independent reservoirs are equally coupled to the localized spin, each will individually attempt to screen the spin, resulting in the formation of a highly-correlated electron state. (c) Physically separating two reservoirs does not suffice to make them independent. If a localized electron can hop off the site to the right reservoir and a new electron can hop onto the site from the left, the two reservoirs will cooperate in screening the localized spin. To create two independent screening channels, processes which transfer electrons from one reservoir to another must be suppressed. (d) Experimental Realization of $2 \mathrm{CK}$. We add an additional finite reservoir (red) to an artificial magnetic impurity connected to an infinite reservoir comprised of two conventional leads (blue). (e) Coulomb blockade suppresses exchange of electrons between the finite reservoir and the normal leads at low temperature. The two reservoirs (blue and red) hence act as two independent screening channels (see Text.)

The system is built from a GaAs/AlGaAs heterostructure containing a low density $\left(n_{e}=2 \times 10^{11} e^{-} / \mathrm{cm}^{2}\right)$, high mobility $\left(\mu=2 \times 10^{6} \mathrm{~cm}^{2} / \mathrm{Vs}\right)$ two-dimensional electron gas (2DEG) $68 \mathrm{~nm}$ below the surface. We follow the proposal by Oreg and Goldhaber-Gordon [17] to produce two independent screening channels for an artificial magnetic impurity. A gate-defined quantum dot containing $\sim 25$ electrons in an area of $0.04 \mu \mathrm{m}^{2}$ plays the role of our magnetic impurity (Fig. I(d), left). Its bare charging energy $U \approx 1 \mathrm{meV}$ and its average single-particle level spacing $\Delta \approx 100 \mu \mathrm{eV}$. Previous experiments which claim to probe $2 \mathrm{CK}$ 10, 11, 12, 13, 14, 15] used a local orbital degeneracy in place of spin, freeing spin of the surrounding conduction electrons to act as the channel index. In contrast, our local degeneracy is a real spin [18], and we use two physically-separated reservoirs (red and blue in Fig. 11d)) for the screening channels. Two leads of the small quantum dot cooperate as a single screening channel with antiferromagnetic coupling $J_{\text {ir }}$ ("infinite reservoir") [19]. An additional lead is made finite in size (red, Fig. 1(d)), so that adding or removing an electron from this reservoir is energetically forbidden at low temperature, a phenomenon known as Coulomb blockade. The area of the finite reservoir is $\sim 3 \mu \mathrm{m}^{2}$, corresponding to a charging energy $E_{c}=100 \mu \mathrm{eV} \approx 1.2 \mathrm{~K}$, and a single-particle level spacing $\Delta_{\mathrm{fr}}=2 \mu \mathrm{eV} \approx 25 \mathrm{mK}$ ("finite reservoir"). This level spacing is only slightly larger than the base electron temperature of our dilution refrigerator $-12 \mathrm{mK}$, as determined by Coulomb blockade thermometry on the small quantum dot - and indeed we cannot resolve these levels even at base temperature. Hence, the finite reservoir has an effectively continuous density of states and can screen the magnetic impurity [20]. Since Coulomb blockade prevents exchange of electrons with the other leads, the finite reservoir acts as a second Kondo screening channel (Fig. 1(e)), with antiferromagnetic coupling $J_{\text {fr }}$, allowing the possibility of observing and studying $2 \mathrm{CK}$. The 2CK Hamiltonian (Eq. (2) ) has three possible ground states, depending on the relative couplings to the two reservoirs: 1CK with the finite reservoir $\left(J_{\mathrm{fr}}>J_{\mathrm{ir}}\right), 1 \mathrm{CK}$ with the infinite reservoir $\left(J_{\mathrm{ir}}>J_{\mathrm{fr}}\right)$, and $2 \mathrm{CK}$ at the quantum critical point $J_{\mathrm{fr}}=J_{\mathrm{ir}}$.

In Fig. 2] we demonstrate that the small quantum dot can act as a tunable magnetic impurity and display the single-channel Kondo effect. If the small quantum dot has an odd number of electrons, it has a net spin and acts as a magnetic impurity. With gate $n$ de-energized $(0 \mathrm{~V})$, the system has three conventional leads (blue and red in Fig. 1), all of which cooperate to screen the magnetic impurity with a single energy scale $k T_{\mathrm{K}}$. At temperature $T \lesssim T_{\mathrm{K}}$ the Kondo effect enhances scattering and hence conductance from one lead to another. We measure the conductance $g \equiv d I /\left.d V_{\mathrm{ds}}\right|_{V_{\mathrm{ds}}=0}$ between the two blue leads. As temperature is increased, the Kondo state is partially destroyed, so 

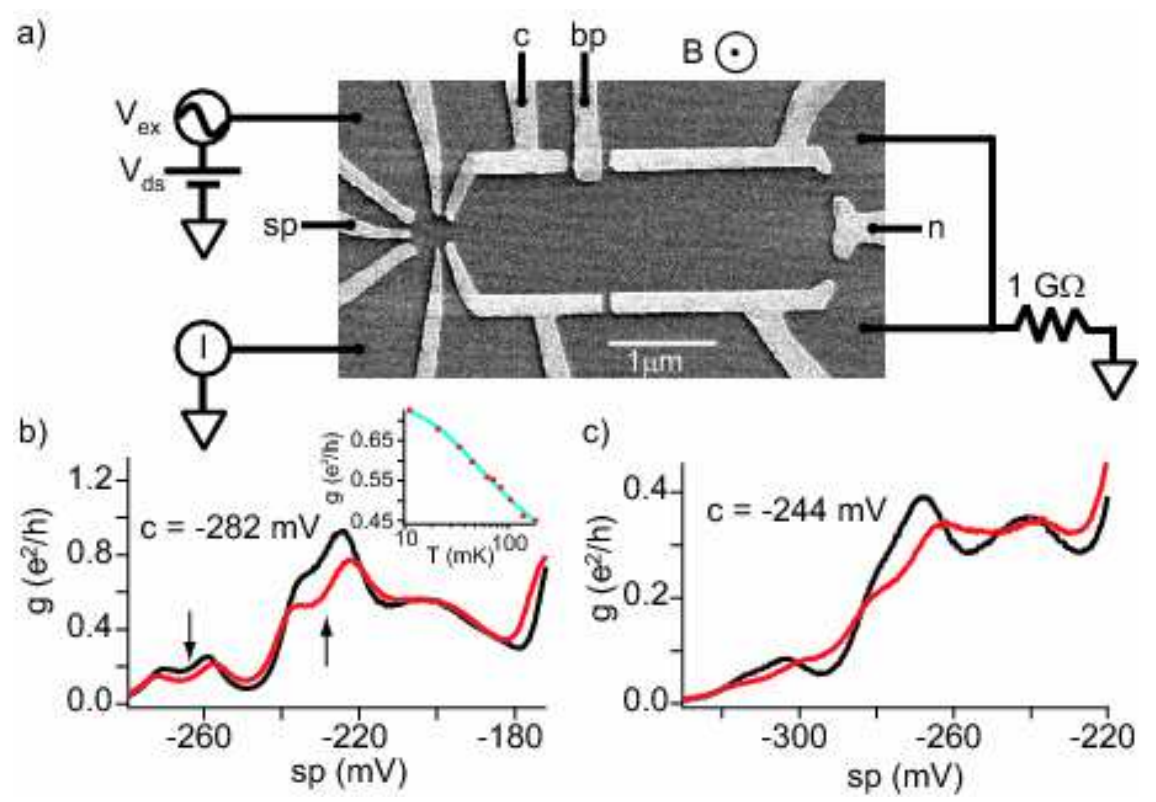

FIG. 2: (a) Scanning electron micrograph of a device similar to that measured. The device consists of a small quantum dot (magnetic impurity, left) coupled to conventional leads (top and bottom left) and to a large quantum dot (finite reservoir, right). Electrons are depleted under every gate by application of negative voltages. In the experiments described here, voltages are varied on gates labeled $c$ ("coupling" between dots), $b p$ ("big dot plunger"), sp ("small dot plunger"), and $n$ ("nose", which opens or closes the big dot). All transport measurements presented in Fig. 3 4 and 5 are measured with source and drain connected as shown, in a magnetic field of $130 \mathrm{mT}$ normal to the plane of the 2DEG. (b) With gate voltage $n=0$, the large dot opens into an infinite reservoir. Arrows mark regions where the small dot has an unpaired spin, leading to enhanced conductance at $12 \mathrm{mK}$ (black) compared to $50 \mathrm{mK}$ (red). Fitting the temperature dependence of the conductance (b inset), we find that Kondo temperature ranges from 110 to $300 \mathrm{mK}$ : see Supplementary Information. In (c), the data from (b) are shown for stronger tunnel coupling to the right lead: $c=-244 \mathrm{mV}$ instead of $-282 \mathrm{mV}$. From temperature dependence of (c), we find that $T_{\mathrm{K}}$ ranges from 180 to $320 \mathrm{mK}$.

the conductance decreases (Fig. 2(b)). This, and all other measurements reported here, are performed in a magnetic field $B=130 \mathrm{mT}$ normal to the plane of the heterostructure. The orbital effect of this modest field suppresses direct transmission through the small quantum dot, which we found to yield Fano lineshapes at zero magnetic field (cf. 22]). Due to the small g-factor of electrons in GaAs/AlGaAs heterostructures, $|g| \lesssim 0.4$, the Zeeman effect of the field is unimportant in both $1 \mathrm{CK}$ and $2 \mathrm{CK}$ regimes - see Supplementary Information for details.

The conductance as a function of temperature (e.g. Fig. 2(b) inset) matches the expected form $\tilde{g}(T)$ for a quantum dot in the Kondo regime, with the addition of a constant offset $a$ :

$$
g(T)=\underbrace{g_{0} f\left(T / T_{\mathrm{K}}\right)}_{\tilde{g}(T)}+a .
$$

$g_{0}<2 e^{2} / h$ reflects the intentionally-imposed asymmetry of coupling to the two conventional leads that comprise the infinite reservoir (see Supplementary Information.) The normalized temperature-dependence of conductance $f\left(T / T_{\mathrm{K}}\right)$ is universal in the Kondo regime - it has no analytic form, but ranges from zero at high temperature to 1 at low temperature, with a broad logarithmic rise around $T=T_{\mathrm{K}}$ 23, 24]. A conductance offset such as we observe has been seen by other experimentalists 25, and is generically expected in the presence of potential scattering 26. As expected, $T_{\mathrm{K}}$ varies strongly across the Kondo valley in both cases, and $T_{\mathrm{K}}$ is higher when the dot is more strongly coupled to the right lead, which increases the total $\Gamma$ of the system. All results presented in this paper are for this same electron occupancy, although we have observed similar behavior in the next Kondo valley (two fewer electrons in small dot), as well as upon thermally cycling the device.

Differential conductance $g\left(T, V_{\mathrm{ds}}\right)=d I / d V_{\mathrm{ds}}$ is enhanced near zero bias (Fig. [3 (b) and (e)) when the electrostatic potential of the small dot is set to the middle of the Kondo valleys in Fig. 2(b) or (c), respectively. This is a manifestation of the enhanced density of states at the Fermi level, widely accepted as one of the classic signatures of the Kondo effect, demonstrating clearly that the small dot acts as a magnetic impurity. Remarkably, the zero bias enhancement changes to zero bias suppression as gate $n$ is made more negative, closing off the big dot to form a finite reservoir with integer occupancy (Fig. [3(f)). The change signals that the single-channel Kondo state with the leads 

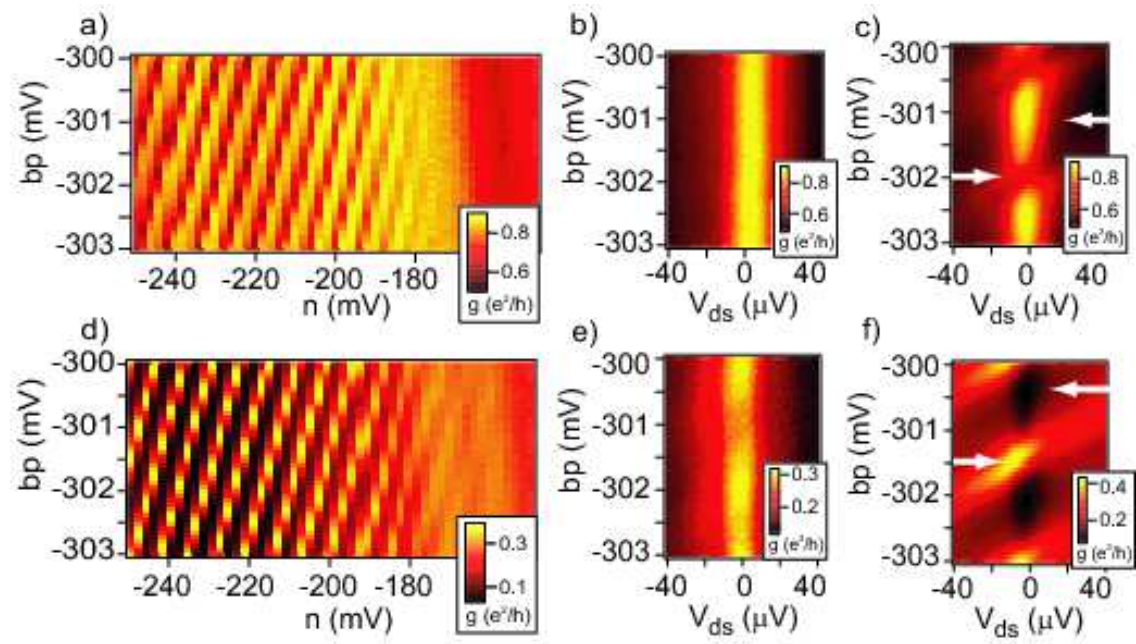

FIG. 3: The formation of two competing 1CK states with two different reservoirs. (a) $s p$ is set so that the small dot has an unpaired spin (middle of a Kondo valley), and $c$ is set to $-282 \mathrm{mV}$. Conductance is shown as a function of $n$ and $b p$. With $n>-180 \mathrm{mV}$, varying $b p$ has little effect on the conductance. As $n$ is made more negative, the reservoir becomes finite and a series of stripes in the conductance map reflects Coulomb blockade in the finite reservoir (see Supplementary Information for explanation of the modulation.) In (b) and (c), differential conductance as a function of bias $\left(V_{\mathrm{ds}}\right)$ and $b p$ is shown for $n=-170 \mathrm{mV}$ and $n=-224 \mathrm{mV}$, respectively. In (b), there is clear zero-bias enhancement, consistent with Kondo effect. In (c), the zero-bias enhancement (denoted by righthand arrow) is modulated by the charge state of the finite reservoir, for reasons explained in the text [2nd paragraph after Eq. (5)]. The lefthand arrow marks the charge degeneracy point of the finite reservoir. (d) Same as (a), except with stronger interdot coupling: $c=-244 \mathrm{mV}$. (e) As in (b), we observe a clear zero-bias conductance enhancement for $n=-170 \mathrm{mV}$. (f) However, in this case at $n=-224 \mathrm{mV}$ the zero-bias enhancement is replaced by zero-bias suppression (denoted by righthand arrow). Here the local spin forms a Kondo state with the finite reservoir, suppressing conductance through the small quantum dot (see Text.) Again, the zero-bias feature is modulated by the charge state of the finite reservoir, and the lefthand arrow marks the charge degeneracy point of the finite reservoir.

has been broken, to form instead solely with the finite reservoir. This occurs for $J_{\mathrm{fr}}>J_{\mathrm{ir}}$, as shown in more detail in Fig. 4 below. With slightly weaker coupling to the finite reservoir (Fig. 3 (c), $J_{\text {ir }}>J_{\text {fr }}$, the Kondo state is formed solely with the infinite reservoir. This effect requires that the finite reservoir have integer occupancy, i.e. the device must be set to a Coulomb blockade valley of the finite reservoir.

In Fig. [4 we provide further evidence that, with the finite reservoir formed, two independent 1CK states can exist depending on the relative coupling of the small dot to the two reservoirs. We have fine control over the occupancy of both the finite reservoir and the small dot with gates $b p$ and $s p$, as shown in Fig. 目(a) and more completely in Supplementary Information. Conductance $g(T) \equiv g(0, T)$ at weak coupling to the finite reservoir $\left(J_{\mathrm{ir}}>J_{\mathrm{fr}}\right)$ fits the

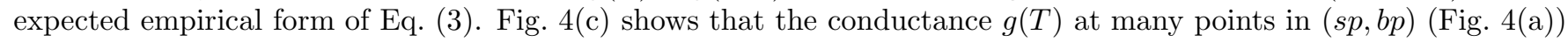
can be collapsed onto a universal curve. The differential conductance $g\left(V_{\mathrm{ds}}, T\right)$ of a $1 \mathrm{CK}$ system is further expected to follow a specific form as a function of both bias and temperature 27], at an energy scale substantially below $k T_{\mathrm{K}}[28]$ :

$$
\frac{g(0, T)-g\left(V_{\mathrm{ds}}, T\right)}{T^{\alpha}}=\kappa\left(\frac{e V_{\mathrm{ds}}}{k T}\right)^{2},
$$

where the exponent $\alpha=2$ is characteristic of $1 \mathrm{CK}$, and $\kappa=0.82 \frac{g_{0}}{T_{K}^{2}}$. The numerical prefactor of order unity is dependent on the underlying model, numerical calculations, and proximity to the symmetric 2CK fixed point (see Supplementary Information), so we simply treat $\kappa$ as a free fitting parameter for each set of gate voltages. Fig. 4(d) demonstrates excellent $1 \mathrm{CK}$ scaling at temperatures of $12,24,28$, and $38 \mathrm{mK}$, all well below $T_{\mathrm{K}}$. A nonlinear fit to the data in Fig. 目(d) yields $\alpha=1.72 \pm 0.40$ (95\% CL), consistent with $\alpha=2$.

In Fig. $4(\mathrm{e}-\mathrm{h})$, we demonstrate that at stronger coupling to the finite reservoir $\left(J_{\mathrm{fr}}>J_{\mathrm{ir}}\right)$ the small dot forms a Kondo state with the finite reservoir, as manifested by low-energy suppression rather than enhancement of conductance between the normal leads of the small dot. We must modify the form we use to fit the temperature dependence to reflect this inversion:

$$
g(T)=\underbrace{g_{0}\left(1-f\left(T / T_{\mathrm{K}}\right)\right)}_{\tilde{g}(T)}+a .
$$



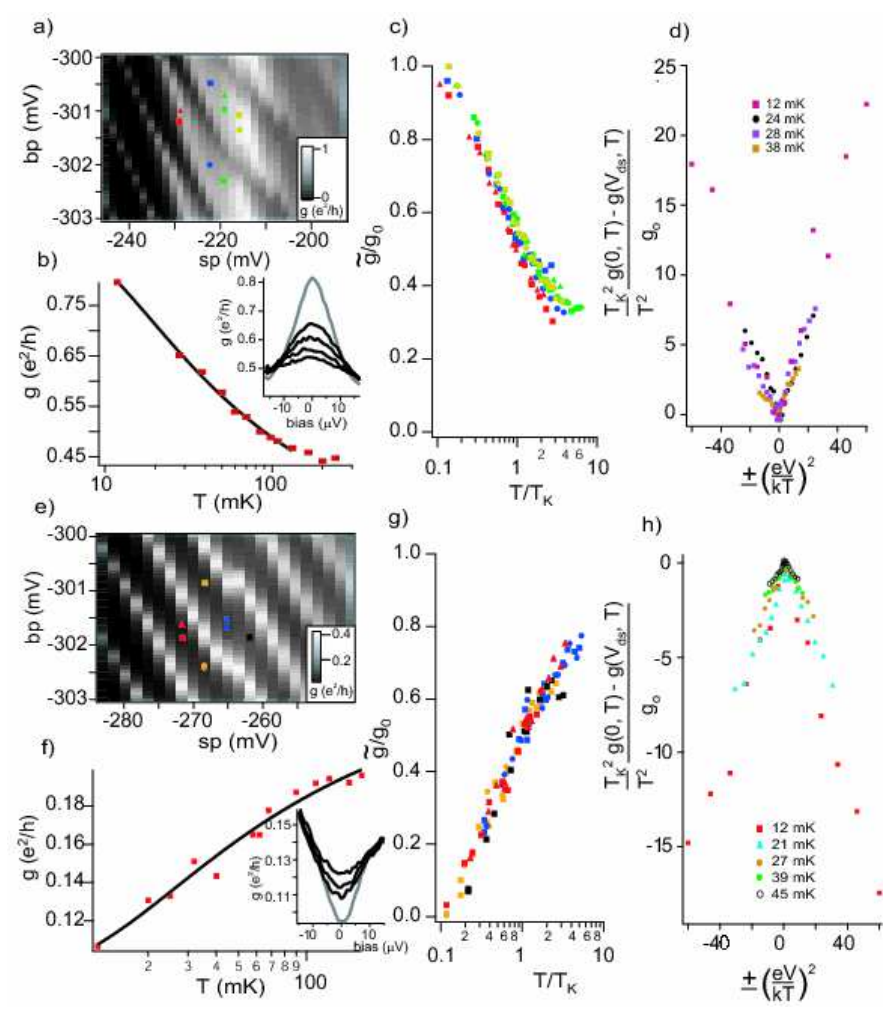

FIG. 4: Energy dependence of Kondo effect, with the finite reservoir formed. (a) - (d) Antiferromagnetic coupling to the infinite reservoir (normal leads) is stronger than coupling to the finite reservoir: $c=-282 \mathrm{mV}$. (a) Conductance as a function of gates $s p$ and $b p$, at $V_{\mathrm{ds}}=0$. (b) Any point in $(s p, b p)$ such that the occupancy of the small dot is odd and that of the finite reservoir is integer shows enhanced conductance at low temperature and low bias (inset: $12 \mathrm{mK}$, grey, to $60 \mathrm{mK}$ ), and a temperature dependence consistent with Kondo effect. (c) The normalized dependence of conductance on temperature is uniform for different points in $(s p, b p)$ space, while $T_{\mathrm{K}}$ ranges from $50 \mathrm{mK}$ to $180 \mathrm{mK}$. $\tilde{g} \equiv g-a$ is the conductance with the temperature-independent offset subtracted off. (d) Plotting a specific combination of temperature and bias collapses the data for a single point in $(s p, b p)$ space $\left(T_{\mathrm{K}}=175 \mathrm{mK}, g_{0}=0.75 e^{2} / h\right)$ onto a single V-shaped curve, corresponding to the scaling relation predicted for $1 \mathrm{CK}$ (Eq. (4)). (e) - (h) Kondo effect with the finite reservoir: $c=-244 \mathrm{mV}$. (e) Conductance as a function of $s p$ and $b p$ : conductance is now suppressed rather than enhanced at low bias and temperature (cf. Fig. 3 (f).) (f) Fitting the conductance as a function of temperature to the empirical form we expect for 1CK with the finite reservoir (Eq. 3 we find that $T_{\mathrm{K}}$ ranges from $30 \mathrm{mK}$ to $130 \mathrm{mK}$. (g) We again normalize and collapse the temperature dependence at several points in $(s p, b p)$ onto a single curve. (h) We collapse differential conductance data like those in inset (f) (12 mK, grey, to $30 \mathrm{mK})$ at a single point in ( $s p, b p$ ) onto a single inverted $\mathrm{V}$-shaped curve using the same temperature-bias scaling as in (d). Deviations from perfect scaling may be related to the slightly lower Kondo temperature $\left(T_{\mathrm{K}}=120 \mathrm{mK}, g_{0}=0.16 e^{2} / h\right.$.

Again temperature dependence at multiple points in $(s p, b p)$ (Fig. 4(e)) collapse onto a single normalized Kondo form $\tilde{g} / g_{0}$ vs $T / T_{\mathrm{K}}$ (Fig. 目(g)), providing strong evidence that a distinct $1 \mathrm{CK}$ state has formed with the finite reservoir. Furthermore, using the same scaling relation as above (Eq. (44) the data again collapse onto a single (inverted) curve at low bias and temperature (Fig. U(h)). Interestingly, we find the numerical prefactor of $\kappa$ to be 0.25 in both 1CK regimes - precisely matching each other but only roughly agreeing with our predicted value of 0.82 .

Having established the existence of two distinct Kondo ground states - depending on the ratio $J_{\text {ir }} / J_{\text {fr }}$ - we next demonstrate the tunability necessary to reach the symmetric $2 \mathrm{CK}$ state, $J_{\mathrm{ir}} \approx J_{\mathrm{fr}}$. By setting the tunnel coupling to the finite reservoir to an intermediate value, we can observe either zero-bias enhancement or zero-bias suppression (marked by white arrows in Fig. 5(a)), in both cases away from any charge degeneracy point of the finite reservoir (marked by a black arrow in Fig. 5(a).) This is expected 17], since the antiferromagnetic coupling to a reservoir depends not only on a tunneling rate but also on the energy required to transfer an electron from the local site to that reservoir. Gate $b p$ tunes that addition energy for the finite reservoir, modifying $J_{\text {fr }}$ while keeping $J_{\text {ir }}$ nearly constant. The region in $(s p, b p)$ of suppressed conductance (red) grows rapidly with increasing coupling to the finite reservoir, as seen in Fig. 國(b, c, and d) for $c=-258,-256,-254 \mathrm{mV}$, respectively.

The evolution of $d I / d V_{\mathrm{ds}}$ from zero-bias enhancement to zero-bias suppression as a function of coupling gate $c$ may be seen most clearly in Fig. 国(e). We identify the curve for $c=-260 \mathrm{mV}$ as being very close to the $2 \mathrm{CK}$ symmetric point. At first blush, it is surprising that this curve does not display a clear cusp at low $V_{\mathrm{ds}}\left(G \sim\right.$ const $\left.-\sqrt{V_{\mathrm{ds}}}\right)$. 

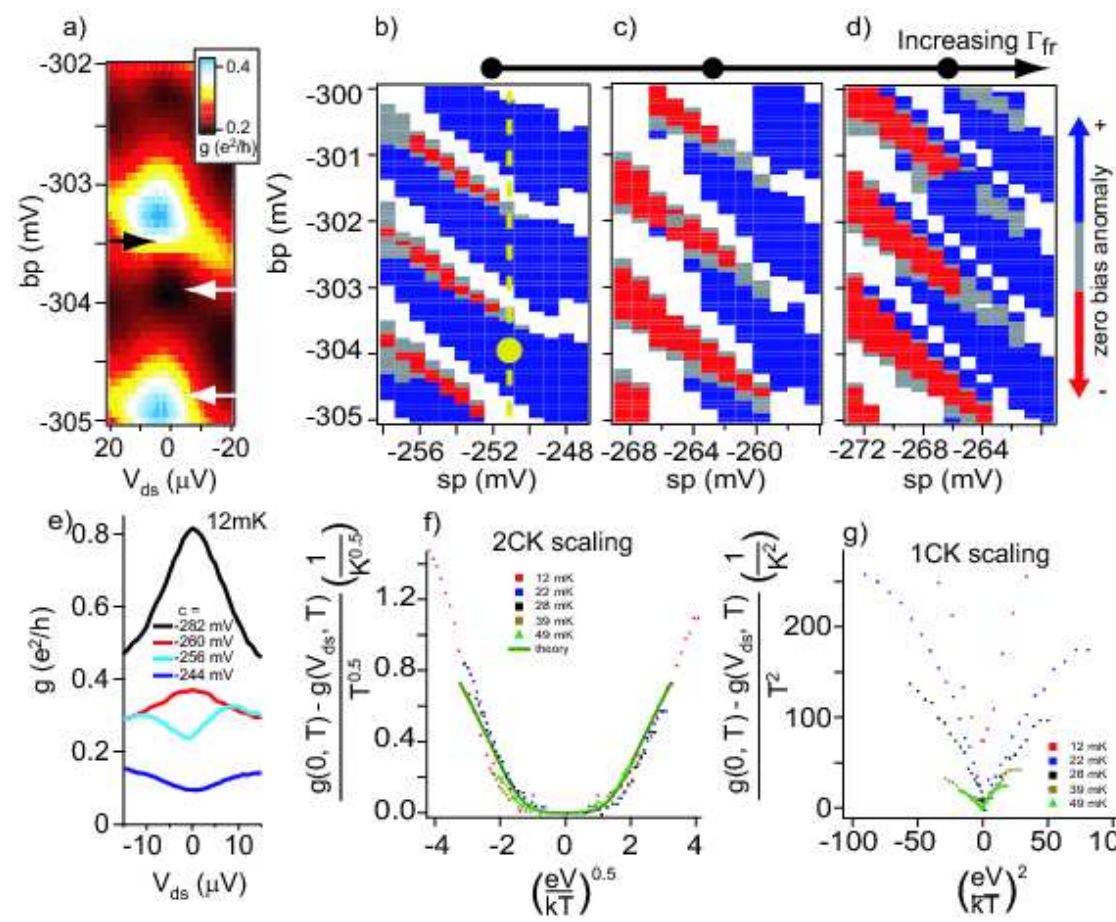

FIG. 5: Evidence for 2CK physics. (a) Differential conductance as a function of $b p$ and $V_{\mathrm{ds}}$ in the middle of a Kondo valley, with intermediate coupling to the finite reservoir $c=-258 \mathrm{mV}$. In contrast to Fig [3 (c) and [3 f), here we observe both zero-bias enhanced and zero-bias suppressed conductance (marked by bottom and top white arrows, respectively) by fine-tuning $b p$. The black arrow marks the charge degeneracy point of the finite reservoir. (b)-(d) At $c=-258,-256$, and $-254 \mathrm{mV}$, conductance may be either enhanced or suppressed at zero bias, depending on the fine tuning of the electrostatic potentials of the small dot and finite reservoir with gates $s p$ and $b p$, respectively. Red and blue indicate regions of suppressed conductance or enhanced conductance, respectively, while gray indicates relatively flat conductance around $V_{\mathrm{ds}}=0$. White regions are too close to charge degeneracy of dot or finite reservoir to diagnose Kondo-induced enhancement or suppression of conductance. Increased coupling to the finite reservoir expands the region of suppressed conductance (red). The yellow dashed line in (b) shows the setting of $s p$ used in (a), and the yellow dot shows the approximate location in the charging hexagon of the symmetric $2 \mathrm{CK}$ point analyzed in (f) and (g). (e) Differential conductance near zero bias evolves with coupling $c$ from zero-bias enhancement to zero-bias suppression. For each curve, $s p$ sets the small dot in the middle of a Kondo valley and $b p$ sets the finite reservoir midway between two charge degeneracy points. (f,g) Tuning $b p$ near $-304.7 \mathrm{mV}$ (bottom white arrow in (a)), we observe that differential conductance depends on bias and temperature with $\alpha=0.5$, consistent with 2 CK (f) and inconsistent with $1 \mathrm{CK}$ (g, which attempts to apply scaling to exactly the same data, but with $\alpha=2$.) In the Supplementary Information we show the converse, namely that in the $1 \mathrm{CK}$ region the $2 \mathrm{CK}$ scaling law does not fit, while the $1 \mathrm{CK}$ scaling does. A two-dimensional nonlinear fit to the data set used for (f) and (g) yields $\alpha_{2}=0.62 \pm 0.21$, consistent with $\alpha=0.5$. In (f) and (g) we do not scale the vertical axis by $T_{\mathrm{K}}, T_{2 \mathrm{CK}}$, or $g_{0}$ because here we lack independent measures of these parameters.

In fact, conformal field theory predicts that at the $2 \mathrm{CK}$ symmetric point the differential conductance should depend quadratically on bias for $e V_{\mathrm{ds}}<k T$, and should only cross over to $\sqrt{V_{\mathrm{ds}}}$ behavior at higher bias $e V_{\mathrm{ds}} \gtrsim 3 k T$ (see green curve in Fig. 5(f)). Such a crossover is hard to see in a single plot of differential conductance versus bias. Instead, we combine the dependence of differential conductance on both bias and temperature in a scaling plot to produce compelling evidence for $2 \mathrm{CK}$. The expected scaling form is somewhat different from that for 1CK [ $\underline{8}, 14$, 27, 29]:

$$
\frac{g(0, T)-g\left(V_{\mathrm{ds}}, T\right)}{T^{\alpha_{2}}}=\kappa_{2} Y\left(\frac{e V_{\mathrm{ds}}}{k T}\right) .
$$

Here $\alpha_{2}=0.5, \kappa_{2}=\left(g_{0} / 2\right)\left(\pi / T_{2 \mathrm{CK}}\right)^{\alpha_{2}}$, and

$$
Y(x)=1-F_{2 C K}(x / \pi) \approx\left\{\begin{array}{cc}
\frac{3}{\pi} \sqrt{x}-1 & \text { for } \quad x \gg 1 \\
c x^{2} & \text { for } \quad x \ll 1
\end{array}\right.
$$

with $c \approx 0.0758\left[30\right.$. As with $\kappa$ in the $1 \mathrm{CK}$ analysis, in practice we treat $\kappa_{2}$ as a free parameter for each set of gate voltages. $F_{2 \mathrm{CK}}$ is found by conformal field theory $[8,31,32$.

Figure 5f f) shows that when we tune close to the $2 \mathrm{CK}$ symmetric point $(c=-258 \mathrm{mV}$ rather than $-260 \mathrm{mV}$, due to a small shift in parameters) data at various temperatures and biases collapse onto each other and match the conformal 
field theory prediction (Eq. (6) $)$, which is scaled vertically by $\kappa_{2}$. The horizontal axis is plotted as $\left(e V_{\mathrm{ds}} / k T\right)^{0.5}$, to emphasize that $g\left(V_{\mathrm{ds}}\right) \sim$ const $-\sqrt{e V_{\mathrm{ds}}}$ for $e V_{\mathrm{ds}} / k T \gg 1$.

In Fig. 5 (g) we show the same data scaled as would be appropriate for $1 \mathrm{CK}$ (Eq. (4) ) instead of 2CK. As anticipated, this scaling fails completely: scaled data for different temperatures deviate from each other even near zero bias. A

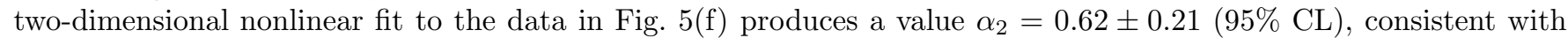
$2 \mathrm{CK}$ behavior. Naively, we would expect $2 \mathrm{CK}$ behavior to persist only up to $\left\{k T, e V_{\mathrm{ds}}\right\} \sim\left(k T_{\mathrm{K}}\right)^{2} / E_{C} \approx 1.7 \mu \mathrm{eV}[33]$. Empirically, 2CK persists to much higher energies: conductance follows the $2 \mathrm{CK}$ scaling form up to $V_{\mathrm{ds}}=15 \mu \mathrm{eV}$, corresponding to $T=180 \mathrm{mK}$, even higher than $T_{K}$. Enhancement of $2 \mathrm{CK}$ energy scales has been predicted in our geometry in the presence of charge fluctuations [34], but is not expected to be so dramatic for our parameter values.

In this Article, we have presented data demonstrating the existence of two independent 1CK states, along with a study of the associated 2CK state. Remarkably, the conductance of the symmetric 2CK state matches not only a simple power law but rather a complete theoretically calculated non-Fermi-liquid scaling function over a broad range of energy (Eq. 7, Fig. 5(f)). In future, it would be interesting to extend this scaling form theoretically and experimentally to cover the effects of a Zeeman field and slightly asymmetric coupling to the two reservoirs, for example to quantitatively describe the family of curves in Figure 5 (f). Finally, other parameter regimes of the same structure may show additional exotic behavior [18].

We thank A. Schiller, E. Lebanon, F. Anders, I. Affleck, T. Costi, L. Glazman, K. Le Hur, C. Marcus, M. Pustilnik, E. Sela, J. von Delft, and G. Zarand for discussions. EL and FA also performed NRG calculations which gave us crucial intuition regarding where we were in parameter space. Scott Roy helped us understand how to perform nonlinear fits to determine the exponents $\alpha, \alpha_{2}$ for the energy dependence in both $1 \mathrm{CK}$ and $2 \mathrm{CK}$ regimes. This work was supported by NSF CAREER Award DMR-0349354, US-Israel BSF Award \#2004278, DIP and ISF. DGG acknowledges Fellowships from the Sloan and Packard Foundations, and a Research Corporation Research Innovation Award. RMP was supported by an ARO Graduate Fellowship during the early stages of this work. Correspondence and requests for materials should be addressed to goldhaber-gordon@stanford.edu.

* Department of Physics, Harvard University, Cambridge, MA, USA; Present Address: Advanced Micro Devices, Austin, TX.

[1] Goldhaber-Gordon, D. et al. Kondo effect in a single-electron transistor. Nature 391, 156-159 (1998).

[2] Nygard, J., Cobden, D. H. \& Lindelof, P. E. Kondo physics in carbon nanotubes. Nature 408, 342 - 346 (2000).

[3] Park, J. et al. Coulomb blockade and the Kondo effect in single-atom transistors. Nature 417, 722 - 725 (2002).

[4] Liang, W. J., Shores, M. P., Bockrath, M., Long, J. R. \& Park, H. Kondo resonance in a single-molecule transistor. Nature 417, $725-729$ (2002).

[5] Kondo, J. Resistance minimum in dilute magnetic alloys. Progress in Theoretical Physics 32, 37 (1964).

[6] Nozières, P. \& Blandin, A. Kondo effect in real metals. Journal de Physique 41, 193 - 211 (1980).

[7] Zawadowski, A. Kondo-like state in a simple model for metallic glasses. Physical Review Letters 45, 211 - 14 (1980).

[8] Affleck, I. \& Ludwig, A. W. W. Exact conformal-field-theory results on the multichannel Kondo effect: single-fermion Green's function, self-energy, and resistivity. Physical Review B 48, 7297 - 321 (1993).

[9] Cox, D. L. \& Zawadowski, A. Exotic Kondo effects in metals: magnetic ions in a crystalline electric field and tunnelling centres. Advances In Physics 47, 599 - 942 (1998).

[10] Cox, D. L. Quadrupolar Kondo effect in uranium heavy-electron materials. Physical Review Letters 59, 1240 - 1243 (1987).

[11] Seaman, C. L. et al. Evidence for non-Fermi-liquid behavior in the Kondo alloy $\mathrm{Y}_{1-x} \mathrm{U}_{x} \mathrm{Pd}_{3}$. Physical Review Letters 67, $2882-2885$ (1991).

[12] Besnus, M. J. et al. Specific-heat and NMR of the Kondo system $\mathrm{YbPd}_{2} \mathrm{Si}_{2}$. Journal of Magnetism And Magnetic Materials 76-7, $471-472$ (1988).

[13] Ralph, D. C. \& Buhrman, R. A. Observations of Kondo scattering without magnetic-impurities - a point contact study of 2-level tunneling systems in metals. Physical Review Letters 69, 2118 - 2121 (1992).

[14] Ralph, D. C., Ludwig, A. W. W., von Delft, J. \& Buhrman, R. A. 2-channel Kondo scaling in conductance signals from 2-level tunneling systems. Physical Review Letters 72, 1064 - 1067 (1994).

[15] Cichorek, T. et al. Two-channel Kondo effect in glasslike ThAsSe. Physical Review Letters 94, 236603 (2005).

[16] In dilute rare earth/actinide alloys, thermodynamic evidence is largely compatible with $2 \mathrm{CK}$, but the inability to reconcile transport data with $2 \mathrm{CK}$ theory has left the final state of understanding inconclusive. In the putative atomic two-level system 2CK measured by Ralph et al. in narrow metal constrictions 13, 14] and more recently by Cichorek et al. in bulk metallic glasses [15], the transport data nicely match the expected scaling behavior, but a fundamental controversy persists about whether the original (near-degenerate but strongly-coupled two-level system) model Hamiltonian can even exist in a real physical system.

[17] Oreg, Y. \& Goldhaber-Gordon, D. Two-channel Kondo effect in a modified single electron transistor. Physical Review Letters 90, 136602 (2003). 
[18] More complex scenarios are possible in different parameter regimes of the same structure we study. These include 2CK in which the local twofold degeneracy is between two charge states rather than two spin states [34], 2CK involving a mixture of spin and charge degrees of freedom [35], and SU(4) Kondo involving both spin degeneracy on the local site and charge degeneracy in the finite reservoir [36].

[19] Glazman, L. I. \& Raikh, M. E. Resonant Kondo transparency of a barrier with quasilocal impurity states. JETP Letters 47, 452-455 (1988).

[20] Thimm, W. B., Kroha, J. \& von Delft, J. Kondo box: A magnetic impurity in an ultrasmall metallic grain. Physical Review Letters 82, 2143-46 (1999).

[21] Haldane, F. D. M. Scaling theory of the asymmetric Anderson model. Physical Review Letters 40, 416-419 (1978).

[22] Göres, J. et al. Fano resonances in electronic transport through a single-electron transistor. Physical Review B 62, 2188-94 (2000).

[23] Costi, T. A. \& Hewson, A. C. Transport coefficients of the Anderson model via the numerical renormalization group. J. Physics: Cond. Mat. 6, 2519-2558 (1994).

[24] Goldhaber-Gordon, D. et al. From the Kondo regime to the mixed-valence regime in a single-electron transistor. Physical Review Letters 81, 5225 (1998).

[25] J. Folk and A. Huettel, private communications.

[26] Pustilnik, M. \& Glazman, L. I. Kondo effect in real quantum dots. Physical Review Letters 87, 216601 (2001).

[27] Understanding conductance at finite bias can require a nonequilibrium treatment [28]. However if the coupling to the two conventional leads that form the reservoir is substantially asymmetric the Kondo resonance is pinned to the fermi level of the strongly-coupled lead and the system is in equilibrium even for finite bias. In our setup we found $\Gamma_{\text {ir } 1 / \Gamma_{\text {ir } 2} \approx 8, \text { so this }}$ equilibrium condition is satisfied.

[28] Majumdar, K., Schiller, A. \& Hershfield, S. Nonequilibrium Kondo impurity: Perturbation about an exactly solvable point. Physical Review B 57, 2991 - 2999 (1998).

[29] Hettler, M. H., Kroha, J. \& Hershfield, S. Nonlinear conductance for the two channel Anderson model. Physical Review Letters 73, $1967-70$ (1994).

[30] Yuval Oreg, unpublished.

[31] Pustilnik, M., Borda, L., Glazman, L. \& von Delft, J. Quantum phase transition in a two-channel-Kondo quantum dot device. Physical Review B 69, 115316 (2004).

[32] von Delft, J., Ludwig, A. W. W. \& Ambegaokar, V. The 2-channel Kondo model. II. CFT calculation of non-equilibrium conductance through a nanoconstriction containing 2-channel Kondo impurities. Annals of Physics 273, $175-241$ (1999).

[33] Florens, S. \& Rosch, A. Climbing the entropy barrier: Driving the single- towards the multichannel Kondo effect by a weak Coulomb blockade of the leads. Physical Review Letters 92, 216601 (2004).

[34] Lebanon, E., Schiller, A. \& Anders, F. B. Enhancement of the two-channel Kondo effect in single-electron boxes. Physical Review B 68, 155301 (2003).

[35] Anders, F., Lebanon, E. \& Schiller, A. Coulomb blockade and non-Fermi-liquid behavior in quantum dots. Physical Review $B$ 70, 201306(R) (2004).

[36] Le Hur, K., Simon, P. \& Borda, L. Maximized orbital and spin Kondo effects in a single-electron transistor. Physical Review B 69, 45326 (2004). 